\title{
Molecular analysis suggests oligoclonality and metastasis of endometriosis lesions across anatomically defined subtypes.
}

Running title: clonality and metastasis in endometriosis

Teresa H. Praetorius ${ }^{1,2}$, Anna Leonova ${ }^{2,3}$, Vivian Lac ${ }^{2}$, Basile Tessier-Cloutier ${ }^{2,4}$, Janine Senz ${ }^{2}$, Tayyebeh M. Nazeran ${ }^{2}$, Martin Köbel ${ }^{5}$, Marcel Grube ${ }^{1}$, Bernhard Kraemer ${ }^{1}$, Paul J. Yong ${ }^{2,3,6}$, Stefan Kommoss ${ }^{1^{*}}$, Michael S. Anglesio ${ }^{2,3^{*}}$

\section{Affiliations:}

1) Department of Women's Health, Tübingen University Hospital, 72076 Tübingen, Germany

2) British Columbia's Gynecological Cancer Research Program (OVCARE), University of British Columbia, Vancouver General Hospital, and BC Cancer, Vancouver, BC, Canada, V5Z 4E6

3) Department of Obstetrics and Gynecology, University of British Columbia, Vancouver, Canada, V6H $3 Z 6$

4) Department of Anatomical Pathology, Vancouver General Hospital, Vancouver, Canada, V5Z $1 \mathrm{M} 9$

5) Department of Pathology and Laboratory Medicine, University of Calgary, Calgary, Canada, T2N 2 T9

6) BC Women's Centre for Pelvic Pain \& Endometriosis, BC Women's Hospital and Health Centre, Vancouver, Canada, V6H 3N1

${ }^{*}$ Corresponding Authors (These authors contributed equally to this work):

Dr. Michael S. Anglesio, PhD

m.anglesio@ubc.ca; University of British Columbia, Robert HN Ho Research Centre; 2660 Oak Street, Vancouver, BC, Canada. V6H $3 Z 6$

Prof. Dr. med. Stefan Kommoss

stefan.kommoss@med.uni-tuebingen.de; Department of Women's Health, Tübingen University Hospital, Calwerstraße 7, 72076 Tübingen, Germany 


\section{Abstract}

2 Endometriosis symptoms are heterogeneous with controversy on

3 whether it constitutes a single disease or multiple distinct types.

4 Our previous work found recurrent somatic cancer-driver

5 alterations in endometriosis; however, these have not been found

6 ubiquitously. A handful of cases spread across studies also

7 suggest mutations might be shared (clonal) between lesions of the

8 same type. As current classification systems correlate poorly with

9 symptoms or outcomes, somatic genomics may improve the

10 current system. Here, we investigate heterogeneity of somatic

11 cancer-driver mutations within patients and across endometriosis

12 types.

13 We examined anatomically distinct types of endometriosis

14 (ovarian, deep infiltrating, and superficial endometriosis) in 27

15 individual patients all of whom had at least two types of

16 endometriosis. Specimens were analyzed using high-sensitivity

17 targeted sequencing with orthogonal validation from droplet digital

18 PCR and mutation-surrogate immunohistochemistry.

19 Results found 13/27 patients had informative somatic driver

20 mutation in endometriosis, $9 / 13$ had identical mutations across

21 distinct lesions. Endometriomas tended to have a potential for

22 higher mutational load, and this often resulted in functionally

23 redundant driver mutations in same gene within the same lesions. 
26 Our data are consistent with clonality across endometriosis

27 lesions regardless of subtype. Further the finding of redundancy in

28 mutations with the same gene and lesions is also consistent with

29 endometriosis representing an oligoclonal disease with

30 dissemination likely to consist of multiple epithelial clones

31 travelling together. this suggests the current anatomically defined

32 classification of endometriosis does not fully recognize the

33 etiology of the disease. A novel classification should take into

34 account genomic and other molecular features. These findings

35 could further contribute to development of a more personalized

36 endometriosis diagnosis and care.

\section{Introduction}

39 Endometriosis is a chronic estrogen-dependent inflammatory

40 disease defined by the presence of endometrial epithelial glands

41 and stroma outside the uterine lumen and a small but significant

42 risk of malignant transformation (1-3). It is estimated to affect up to

$4310 \%$ of biological females of reproductive age and can result in

44 severe adverse effects on quality of life and productivity $(4,5)$.

45 Clinical symptoms of endometriosis are diverse and include pelvic

46 pain, dysmenorrhea, dyspareunia, and infertility $(2,6)$. Three

47 major anatomically described types of endometriosis are

48 recognized: superficial peritoneal endometriosis (EM), deep

49 infiltrating endometriosis (DIE) and ovarian endometriomas

50 (OMA). In addition, rare types such as mass forming/tumorous or 
51 iatrogenic forms also exist. Current classification systems such as

52 the revised American Society for Reproductive Medicine (rASRM)

53 scoring system and the ENZIAN classification for DIE are useful in

54 documenting surgical findings in a standardized manner.

55 However, they poorly correlate with the severity of symptoms and

56 fail to provide a prognostic tool concerning the treatment outcome

57 for pain or infertility $(7,8)$. Likewise, classification and staging of

58 endometriosis are based on clinical findings and surgical

59 presentation and do not include any information about the

60 molecular potential or microenvironment of these lesions.

61 Recent studies have now shown that multiple forms of

62 endometriosis harbor somatic cancer-driver alterations including

63 recurrent activating changes in KRAS, PIK3CA, ARID1A and

64 others (9-11). While it appears the underlying malignant potential

65 for the vast majority of lesions, despite the presence of recurrent

66 driver mutations, remains low (6), their ultimate function in the

67 pathobiology of endometriosis remains unclear. These findings

68 are particularly important to monitoring disease since somatic

69 alteration could be therapeutically targeted or tracked to examine

70 etiology and potential disease dissemination. The concept that

71 endometriosis disseminates is not novel. Endometriosis frequently

72 presents with multiple anatomical sites affected by lesions(12, 13);

73 recent findings confirm a high rate of coexistence between

74 OMA, EM, and DIE where only $2.3 \%$ of study had isolated

75 OMA (12). Along with current etiology largely attributing an 
76 origin to endometrial tissues, all endometriosis lesions may well

77 have disseminated from a eutopic point of origin. This highlights

78 the importance of studying the mechanisms of clonal

79 dissemination and metastasis of endometriosis $(11,13,14)$.

80 Nonetheless, there is little objective evidence in support of

81 widespread clonal dissemination of endometriosis tissues.

82 Clinical presentation of endometriosis is heterogeneous and it is

83 controversial whether endometriosis constitutes one disease entity

84 or whether independent types with different underlying

85 pathogenesis exist (15). As noted above somatic alteration could

86 be used to examine the spread and distribution of distinct

87 endometriosis clones within a given individual. Here we explored

88 the potential clonal relationship of endometriosis from 27 patients

89 with multiple anatomically separated lesions, and each having at

90 least two distinct types of endometriosis. Our initial objective was

91 assessment of clonality at the level of endometriosis anatomically

92 described types, asking questions such as: are mutations

93 frequently shared between lesions and/or between lesion types. If

94 they are not, this would suggest each anatomically defined type

95 represents a unique disease. If they are this would indicate

96 plasticity between types and/or that our understanding of

97 endometriosis types is currently insufficient. 
100 Experimental subject details

101 Formalin-fixed and paraffin-embedded (FFPE) archival tissues

102 from 27 patients from the Tübingen University Hospital, Germany

103 were included. Inclusion criteria included histopathological

104 diagnosis of endometriosis, presentation of two or more types of

105 endometriosis (deep infiltrating, and/or superficial, and/or ovarian

106 endometrioma) in distinct anatomical locations, and lesions

107 estimated to be of sufficient size for macrodissection. Patients with

108 a history of, or co-existing, gynecological cancer were excluded.

109 Experiments were done at the University of British Columbia and

110 the University of Calgary. The project was conducted in

111 compliance with the Canadian Tri-Council Policy Statement on

112 Ethical Conduct for Research Involving Humans (TCPS2, 2018),

113 effort to obtain written informed consent was exercised for all

114 patients. Specimen from non-contactable patients (lost

115 contact/deceased) treated more than 5 years before the start of

116 the study were included under institutionally approved waiver of

117 consent (Tübingen University Hospital Research Ethics Board). All

118 institutions approved use of materials and associated clinical data

119 through local research ethics boards.

120 Sample processing and DNA extraction

121 FFPE specimens were sectioned onto glass slides, stained with

122 dilute hematoxylin and eosin, and manually enriched for

123 endometriosis glands and stroma by manual microdissection as

124 described previously $(10,16)$. DNA was extracted using the 
125 ARCTURUS $\AA$ PicoPure ${ }^{\circledR}$ DNA Extraction Kit (ThermoFisher

126 Scientific, USA) and quantitated using the Qubit 2.0 Fluorometer

127 (ThermoFisher Scientific, USA).

$128 \quad$ Targeted sequencing

129 DNA (45-75ng) was sequenced using a proprietary hypersensitive

130 cancer hotspot sequencing panel: FIND IT ${ }^{\mathrm{TM}}$, version 3.4 (Canexia

131 Health, Canada) $(9,10)$. This assay includes hotspots from 33

132 genes $(10,16)$ (Table S1). Mutations were considered "true", if

133 they were genuine hotspot mutations targeted by the FIND IT

134 assay and previously reported in the Catalogue of Somatic

135 Mutations in Cancer (COSMIC) (17), as well as prior observations

136 with validation $(9,10)$, including a probability score $>0.8$ and a

137 variant allele frequency $(\mathrm{VAF})>0.8 \%$ (see Table S2A).

$138 \quad$ Validation via droplet digital PCR

139 Somatic mutations identified by targeted sequencing were

140 orthogonally validated through droplet digital polymerase-chain-

141 reaction (ddPCR) assays. In addition, selected alterations were

142 tested by ddPCR in all available lesions from a given patient if

143 they were observed either in any one lesion from a given patient

144 (subjected to the FIND IT panel assay), regardless of the results

145 obtained in other panel-tested lesions from the same patient and

146 including any specimens from the same patient that were initially

147 omitted from panel testing due to low DNA yield. Using previously

148 established methods $(10,16)$, extracted DNA was pre-amplified

149 for targets over 10 cycles then diluted before assembling the 
150 ddPCR assay. Droplets were generated using the QX200 Droplet

151 Generator (Bio-Rad Laboratories, USA), amplified by thermal

152 cycling, and quantified using the QX2000 Droplet Reader (Bio-

153 Rad Laboratories, USA). As above alterations were considered

154 "true" if they passed the above noted probability and VAF

155 thresholds for targeted sequencing or were detected using ddPCR

156 above a droplet count set at the average of the negative control

157 specimens plus 3 times the standard deviation of negative

158 controls the relevant assay. See Table S3 for primer/probe details.

159 ARID1A, PTEN and p53 Immunohistochemistry

160 Immunohistochemistry (IHC) assays for ARID1A (18), p53 (19,

$16120)$ and PTEN $(10,16)$ (Table S3) were used as surrogate for

162 somatic alterations following established standards for staining

163 and scoring. ARID1A was stained on Dako Omnis automated

164 immunostainer (Agilent Technologies, USA) or Ventana

165 BenchMark Ultra autostainer (Ventana Medical Systems, USA)

166 the ARID1A rabbit polyclonal antibody, HPA005456 (Sigma-

167 Aldrich). PTEN was stained on Ventana Discovery Ultra (Ventana

168 Medical Systems, USA) using the rabbit monoclonal antibody,

169 138G6 (Cell Signaling, USA). p53 was stained on Dako Omnis

170 (Agilent Technologies, USA) using the p53 mouse monoclonal

171 antibody DO-7 (GA61661-2; Agilent Technologies, USA). Slides

172 were scored by pathologists TMN, and/or BTC, and/or MK.

173 Calling Criteria 
174 Calling criteria was applied to all detected alterations as noted

175 above, including a probability score $>0.8$ and VAF $>0.8 \%$ for panel

176 sequencing and individual droplet count thresholds for each

177 ddPCR assay $(10,16)$.

178 Immunohistochemistry assays were performed and scored

179 according to established standards, briefly ARID1A loss if nuclear

180 staining was absent in endometriosis epithelium cells and internal

181 control (stroma) was intact (18). Mutant p53 (p53abn) if high

182 intensity positive staining was observed in 10 or more adjacent

183 cells in the epithelial cyst wall of endometriosis or complete

184 absence of staining in the entire cyst wall, while maintaining a

185 normal type pattern in surrounding tissue $(19,20)$. PTEN loss if

186 cytoplasmic and nuclear staining was absent in endometriosis

187 epithelium cells and internal control (stroma) was intact $(10,16)$.

188 Statistics

189 Student's t-test was performed to compare the affected genes and

190 lesion types. However, given our limited sample size, they

191 remained non-significant.

193 Results

194 We examined 73 endometriosis lesions from 27 patients with a

195 mean age of 34.9 years (23-45 years; Table S2; Figure S1). 53

196 lesions were subjected to panel-based sequencing with validation

197 of selected alteration by ddPCR, 6 additional samples included in 
198 ddPCR validation only. In cases with sufficient material, lesions

199 were also subjected to mutation surrogate IHC for PTEN (66

200 interpretable), ARID1A (66 interpretable) and p53 (47

201 interpretable). A total of 27/59 (45.8\%) lesions from 13/27 (48\%)

202 cases were confirmed to have identifiable somatic cancer-driver

203 mutations. It should be noted that lesion with mutations observed

204 near, but below, our positive detection thresholds were considered

205 negative, as were alteration which appeared above threshold in

206 panel sequencing data but failed to exceed detection threshold in

207 ddPCR. For example, in a $14^{\text {th }}$ case panel testing suggested

208 alteration but these fell below detection threshold at the ddPCR

209 stage (case 7; Table S2). Nonetheless, potential interpretation for

210 sub-clonal/sub-detection threshold alterations is discussed in a

211 limited context where relevant.

212 Amongst the panel screened lesions, we found nearly half of the

213 tested cases had at least one mutation; where identical assays

214 were applied, PIK3CA alterations were the most common (27

215 hotspot mutations, affecting 12/53 lesions in 6 cases), followed by

$216 K R A S$ (16 hotspot mutations, affecting 15 lesions in 6 cases) and

217 CTNNB1 (10 hotspot mutations, affecting 7/53 lesions in 4 cases;

218 Figure 1). In contrast to the overall frequency of alterations more

219 lesions were affected by KRAS alteration overall. This trend was

220 the same regardless of lesion type (Figure 1D) or if summarizing

221 by fraction of affected cases (Figure 1E). Further, OMA tended to

222 have a higher proportion of lesions affected by somatic cancer- 
223 driver alterations and subsequently higher mutation load than

224 other lesion types (Figure 1, Table S2).

225 Alterations were less frequently observed in NRAS (6 mutations,

226 6/53 lesions, 4 cases), ERBB2 (2 mutations, 2/53 lesions, 1 case)

227 and EGFR (2 mutations, 2/53 lesions, 2 cases). Although our

228 cohort had insufficient numbers to support strong associations, we

229 noted many lesions, predominantly amongst OMA, contained

230 multiple mutations in the same genes. OMA also tended to have

231 higher mutational burden on average (amongst our screened

232 panel mutations). No samples had identifiable p53-abnormal

233 staining pattern (0/47 lesions), PTEN loss was observed in 5

234 cases (8/66 lesions), and ARID1A loss was observed in 1 case

235 (2/66 lesions).

236 Immunohistochemical data is inconclusive with respect to

237 clonality, and similar abnormal patterns were considered in

238 support of mutation findings. Our mutation data suggested a

239 single TP53 alteration but no abnormal p53 pattern was observed

240 by $\mathrm{IHC}$, we considered this as a false positive (case 22; Table

241 S2A). We observed no PTEN mutations in sequencing data

242 despite evidence of loss in PTEN protein expression by $\mathrm{IHC}$ in

243 8/66 lesions (Table S2B), however only a small number of

244 hotspots are covered in panel sequencing. In one case (case 2)

245 with PTEN loss in multiple lesions other somatic (clonal) point

246 mutations were shared between the PTEN-loss affected lesions

247 (Table 1; Table S2A). Case 3 showed loss of ARID1A protein 
248 consistent with loss-of function somatic mutation (18) in both an

249 EM and OMA samples from this patient (Figure 2). Unfortunately,

250 insufficient tissue was available to validate any mutation in the EM

251 sample, however, the PIK3CA (p.Met1043lle) and KRAS

252 alterations were shared across all other lesions from this case.

253 Altogether we observed clonality between at least two

254 endometriotic lesions in 8 out of 13 informative cases (Table S2A;

255 Figure S2).

256 For ease in presenting results details we have divided informative

257 cases into two categories. Simple cases, with only one or two

258 altered genes and/or one or two informative lesions, and complex

259 cases, with larger numbers of alterations across lesions and

260 lesions with functionally redundant mutations.

$261 \quad$ Simple cases

262 We annotated 7 cases as simple (Table 1) based on presence of

263 up to 2 alterations and no functionally redundant (i.e. equivalent

264 activating change resulting from different nucleotide and/or amino

265 acid substitution in the same gene) mutations seen in same

266 lesion. We identified and validated shared mutations across

267 multiple lesions and different types in 3 cases (Figure S2). Case 6

268 shared mutations in PIK3CA between EM and DIE. The other two

269 cases shared mutations in KRAS. Case 19 shared a p.Gly12Ser

270 mutation between an OMA and a DIE. Case 21 shared a

271 p.Gly12Asp between an OMA and three different DIE lesions. 
272 In 4 cases mutations were uninformative with respect to clonality

273 (Figure S2), 3 of which had mutations observed in only a single

274 lesion (KRAS or EGFR). In the last (Case 9) we detected different

275 mutations in the lesions, consisting of different KRAS alterations

276 as well as one PIK3CA alteration - none shared between lesions.

\section{Complex Cases}

278 All six cases in this complex category (Table 1) showed evidence

279 of a clonal relationships between lesions and in each case at least

280 one lesion showed multiple functionally redundant hotspot

281 alterations (Figure S2; Figure 2 and 3). In one case we observed

282 identical somatic alterations between EM and DIE lesions (case

283 13), in 4 cases between DIE and OMA (cases 1, 2, 3, 8; Figure 2)

284 and in one case between all three types (case 4; Figure 3).

285 Functional redundancy was most common with PIK3CA, seen in

28612 lesions across 5 cases. Up to 5 simultaneously observed

287 activating changes in PIK3CA were validated in a single lesion

288 (case 2). In 4/5 cases with PIK3CA mutations we further observed

289 co-existing alterations in CTNNB1, where at least one was shared

290 across multiple sites/types within the affected patient. In 3/4 cases

291 redundancy in CTNNB1 was seen with up to 3 simultaneously

292 observed alterations in the same lesion (case 1 OMA; case 2 DIE;

293 and case 4 shared between DIE and EM). The fourth case had

294 only a single observed (PIK3CA) co-existing CTNNB1 alteration

295 shared between an OMA and a DIE (case 8). A single complex

296 case with a PIK3CA alteration had no co-existing CTNNB1 
297 alterations, at least within our panel assay and therefore no further

298 testing by ddPCR was done. A single case (Case 13) lacking

299 PIK3CA alteration did have multiple redundant CTNNB1

300 alterations, with at least one shared between DIE and EM (an

301 OMA specimen from the same case had insufficient material for

302 testing).

303 KRAS alterations were seen in 5 of 6 complex cases, only case 1

304 in this category showed multiple redundant activating changes

305 (p.Gly12Val in DIE, p.Gly12Ser in both DIE and OMA). This

306 particular case also had multiple redundant mutations in PIK3CA

307 (4/4 shared), and CTNNB1 (3/3 shared), as well as an NRAS

308 (shared) alteration. As PIK3CA, CTNNB1 and KRAS alteration

309 were all relatively common we saw co-existence between these in

$3103 / 6$ cases. The single case (Case 13) that lacked PIK3CA

311 alterations had both CTNNB1, EGFR and ERBB2, shared

312 between DIE and EM. A KRAS mutation was detectable in this

313 case only in the DIE.

$314 N R A S$ alteration were detected in 4 of 6 complex cases, no case

315 showed multiple redundant alterations of NRAS itself but overlaps

316 with KRAS mutations were present. In 3 cases (Cases 1, 2, 8) an

317 NRAS alteration was shared between an OMA and a DIE lesion.

318 In case 4 we detected one NRAS alteration shared between an

319 OMA, a DIE lesion and an EM.

$320 E R B B 2$ alterations were detected in 2 of 6 complex cases, one

321 alteration shared between an OMA and two DIE lesions (case 3) 
322 as well as the shared mutation in case 13 as noted above. An

323 EGFR alteration was also shared between the two analyzed

324 lesions in case 13.

325

\section{Discussion}

327 We examined a total of 73 lesions from 27 individuals affected by

328 endometriosis, we focused on within patient heterogeneity - a

329 largely overlooked feature amongst current genomic studies on

330 endometriosis. Cases were specifically selected to have multiple

331 anatomical sites affected as well as multiple "types" of

332 endometriosis including EM, DIE and OMA. Using a high-

333 sensitivity, and error-correcting, sequencing technology followed

334 by validation and additional screening with ddPCR we identified

335 somatic cancer driver alterations in lesions from 13 patients. A

336 total of 27 lesions had at least one driver alteration, 7 lesions had

337 more than 5.

338 We found evidence of identical alteration across lesions in 10

339 individuals, all of which spanned more than one type of

340 endometriosis. The relatively commonplace finding of identical

341 mutations across lesions suggests that at least a subset is clonal,

342 sharing a common ancestor. Given our present data set the

343 order/directionality of dissemination cannot be determined

344 (Figures 2 and 3). We found that in 9 lesions there appeared to be 345 multiple functionally redundant alterations, which is consistent with 
346 individual lesions being oligoclonal. Functionally redundant

347 mutations were seen in PIK3CA, KRAS, and CTNNB1. This

348 extended to 6 of 13 cases with observed somatic alterations. In 3

349 of these cases the redundant alterations were identified in two or

350 more, suggesting dissemination likely involved multiple epithelial

351 clones traveling together.

352 Examining the specific prevalence of mutations across lesions we

353 observed more PIK3CA mutations than any other. Amongst

354 informative cases OMAs tended to have a higher average

355 mutation burden, at least within our panel, with OMA lesions from

356 cases 1, 2, 3 having multiple activating PIK3CA and/or CTNNB1

357 (and others) driving this average mutation burden up. When

358 examining prevalence at a per lesion or per individual level we

359 found more lesions, and more individuals, to be affected by

360 somatic KRAS mutations. Despite a higher mutation burden within

361 a subset of OMAs, DIE appeared to have a wider range of

362 mutations from our panel with EGFR and ERBB2 mutations seen

363 only in DIE.

364 Our study is unique in examination of a modest number of co-

365 existing lesions. Particular strengths include enrichment of

366 endometriosis glands and stroma, the use of proven high-

367 sensitivity and specificity digital sequencing methods, and

368 orthogonal validation for the majority of alterations (21). We

369 elected to be relatively conservative in calling mutations, however,

370 it should be noted that a number of alterations were found to be 
371 just below detection thresholds, if a single assay was done, or

372 both just above on panel sequencing and just below on ddPCR. In

373 such cases we considered alteration to be negative/unvalidated.

374 In sampling tissue for sequencing assays and subsequent ddPCR

375 we took sequential tissue sections from a block for enrichment

376 and analysis, often requiring re-sampling of tissue for validation.

377 Hence, we must consider that in a small population of cells, such

378 as a microscopic glands of endometriosis epithelium, it is possible

379 that the proportion of mutation harboring cells changes as a

380 specimen is sectioned such that it would genuinely yield a fraction

381 of cells below a detection threshold in one sampling and above a

382 detection threshold in another. If we were to be more liberal with

383 assessment of ground-truth mutations our overall conclusion

384 would not change though it would suggest greater complexity and

385 more examples of oligoclonal dissemination throughout our

386 cohort.

387 Potential weaknesses include the use of macrodissection instead

388 of laser capture microdissection. In this context we cannot reliably

389 use the variant allele frequency for anything other than binary

390 presence or absence assessment of somatic alterations. IHC

391 observations suggested somatic alteration were exclusive to the

392 epithelium, similar to prior observation with laser-capture

393 microdissection, though this was not explicitly monitored in

394 sequencing experiments. In addition, we used only a relatively

395 limited panel assay to screen for mutations. Therefore, while we 
396 can make suggestions about potential lineage of samples with

397 informative mutations, we are missing considerable data about the

398 un-analyzed genome. Thus, this should be considered as a

399 minimum estimate of clonality. We cannot strictly conclude that

400 any cells or lesions (within a given patient) that do not share

401 mutations with those found within our panel are of unrelated

402 lineage. Nonetheless, the presence of functionally redundant

403 alterations (multiple KRAS, PIK3CA, and CTNNB1 alterations)

404 does suggest at the very least divergence of subclones within

405 lesions is likely.

406 Broad dissemination and metastasis in general are common

407 features of advanced cancers but are not typical for a benign

408 neoplastic disease. Endometriosis knowingly shares many

409 characteristics with tumors, and it is also associated with the most

410 lethal gynecologic cancer: ovarian carcinomas, where

411 endometriosis is both a risk factor and proven precursor lesion for

412 both endometrioid and clear cell ovarian carcinomas.

413 All major anatomically described types of endometriosis can

414 harbor recurrent somatic cancer-driver alterations. Our findings

415 validate a modest spectrum of driver alterations, as has been

416 described previously $(9,10,22)$. While single cases have been

417 described to share alteration between lesions of the same type $(9$,

418 14), we provide considerable evidence that shared lineage

419 between lesions is common, not only between lesions of the same

420 type, but across anatomically defined lesion types. If we consider 
421 the likelihood of clonal dissemination of endometriosis, then

422 anatomical lesion type descriptions alone are insufficient and

423 should be supplemented with molecular characterizations - albeit

424 which features are clinically useful is not yet established.

425 In recent years there has been a shift away from seeing

426 endometriosis as one disease entity. In addition to biochemical

427 variations, considerable efforts are being applied to identify

428 subtype-specific genomic alterations. This ongoing trajectory of

429 thought has spiked research of endometriosis biomarkers and

430 subtype-tailored treatment. However, here we demonstrate that

431 sampling and comparing lesions from different individuals based

432 solely upon anatomical classification, may yield inadequate

433 results. Our data supports the presence of identical mutations

434 among lesions of the same individual and suggests the clonal

435 dissemination and metastasis mechanism of disease spreading.

436 This underscores the need for molecular classification when

437 designing personalized endometriosis care.

438 To make significant impact the next generation of endometriosis-

439 genomic studies must apply high stringency methods, with

440 appropriate enrichment of tissues, orthogonal validation and/or

441 error correcting sequencing technologies, and most critically be

442 coupled to large clinical data registries conforming to accepted

443 standards in phenotyping data (21) - such as those promoted by

444 WERF-EPHect $(23,24)$. Early studies on somatic genomics of

445 endometriosis have suggested relatively few alterations per lesion 
$446(9,11,22,25)$. However, this work suggests endometriosis may

447 have moderate genomic complexity. Any future study wishing to

448 correlate genomic heterogeneity with the clinical phenotypic

449 spectrum may be complex, it is not sufficiently informative to

450 sample only one lesion per patient for mutation testing. Accurate

451 assessment of somatic mutation profiles may require a significant

452 fraction of lesions are excised and tested at surgery (rather than

453 ablated without biopsy).

454 In addition to the already appreciated complexity of endometriosis,

455 it has now become even more evident that disease presentation is

456 unique and may depend on many factors. This work has also

457 shown that OMAs had the highest mutation burden, while DIE

458 lesions were associated with wider range of mutation. Considering

459 previous work in the field (26-29), this data is consistent with

460 observations that lesion microenvironment may be more

461 permissive for expansion of multiple clones, and hence the higher

462 risk of malignant transformation (30). DIEs might be surrounded

463 by a more restrictive microenvironment conditions resulting in

464 fewer clones and lower malignant potential. As a specific example

465 of clonal expansion, we observed that multiple redundant PIK3CA

466 activating mutation were prevalent in OMAs. These PIK3CA

467 alterations are also common to endometriosis-associated cancers

468 and appear necessary (in combination with ARID1A) for the

469 generation of a representative clear cell ovarian carcinomas

470 model in mice (31), together suggesting they are critical in 
471 underlying malignant potential. Extraovarian microenvironment

472 restriction on malignant potential has been suggested as a

473 potential mechanism for the generally favorable outcomes

474 observed in low-stage synchronous (yet metastatic) endometrioid

475 ovarian and endometrial carcinomas - etiologies tightly linked to

476 endometriosis(32).

477 As we move forward, consideration should be made to determine

478 if patient-wide endometriosis mutation profiles should include

479 ranking of allele frequency, dissemination/spread, clonality across

480 lesions and anatomic types. Phenotyping clinical characteristics

481 could also monitor eutopic endometrium (16) and pathway

482 enrichment across mutations. Intra-lesion spatial heterogeneity

483 (such as in a large OMA or deep nodules) may warrant study

484 especially in relapse/persistent chronic disease and those

485 associated with malignant progression. Simple binary assessment

486 now seems insufficient and modeling with machine learning

487 approaches may be paramount to identify associations between

488 heterogeneous molecular profiles and heterogeneous clinical

489 presentation.

490

\section{Figure Legends}

492 Figure 1: Fraction of cohort affected by somatic hotspot mutations

493 detected in panel sequencing. (A) Overall split of cases with at

494 least one lesions having at least one somatic cancer-driver 
495 alteration. (B) Numbers of detected somatic mutations, split by

496 affected gene, across our entire cohort. (C) Fraction of mutation

497 affected lesions, split by mutation type. (D) Fraction of mutation

498 affected lesions, split by lesion type. (E) Fraction of mutation

499 affected lesions, summarized by fraction of affected cases.

500 Figure 2: Clonal relationships in Case 3 patient presenting with

501 DIE of the vagina, DIE of the cul-de-sac, an OMA of the left ovary,

502 and EM of the rectum. (A) Detected somatic alteration from

503 sequencing and mutation surrogate IHC suggest identical/clonal

504 alteration in KRAS, ERBB2, and PIK3CA (p.Met1043lle) between

505 OMA and two anatomically distinct lesions of DIE. Additional

506 PIK3CA alterations are also visible in the OMA and vaginal DIE.

507 Heterogeneous loss of ARID1A is observed in only the OMA and

508 EM (insufficient material was available for mutation testing in EM).

509 (B) Three possible dissemination models explaining the mutational

510 pattern: (1) top panel illustrates a linear pattern wherein clones

511 from the cul-de-sac travel to the vagina and acquired additional

512 alterations in PIK3CA. Subsequent transfer of all clones to the

513 ovary where subclonal-ARID1A loss occurs. It may further be

514 speculated that the EM resulted from a transfer of clones between

515 the vaginal DIE and OMA (as the initial site for ARID1A loss) or

516 subsequent spread after clones established on the ovary. Given

517 the lack of mutational data on the EM it may also be entirely

518 independent. (2) middle panel illustrates an example where a

519 complex set of clones exist at the ovary and only a subset of these 
520 break-off and colonize vaginal, cul-de-sac and EM/rectal sites.

521 Finally, (3) Lower panel illustrates parallel dissemination from the

522 cul-de-sac lesion to both the vaginal and ovarian sites. Herein we

523 may consider sub-threshold signal from ddPCR of p.Arg88Gln,

524 p.Glu545Lys and p.Gly1049Ser PIK3CA alteration (Table S2) as

525 weak evidence of emerging/undetectable clones that

526 subsequently expand post-transfer of cells to both vagina and

527 ovary. In this model again, the OMA acquires loss-of function

528 ARID1A alterations. Additional possibilities may also explain the

529 mutational patterns. (C-D-E) photomicrographs showing ARID1A

$530 \mathrm{IHC}$ results including regions of loss (surrogate for loss-of function

531 mutation; black arrows) and normal (retained nuclear staining; red

532 arrows) in endometriosis epithelium.

533 Figure 3: Clonal relationships in Case 4 patient presenting with

534 DIE of the rectum, EM of the cul-de-sac and an OMA on the left

535 ovary. (A) Detected somatic alteration from sequencing suggest

536 all variants are shared between DIE and EM lesions. In contrast

537 the ovarian lesion shares only 1 PIK3CA and 1 KRAS alteration

538 with the other two and has no (detected) unique changes. (B)

539 Three possible dissemination models explaining the mutational

540 pattern: (1) top panel illustrates a linear model wherein clones

541 from the ovary travel to the rectum, expand and acquire additional

542 alteration. Cells from the DIE containing all clones then seed the

543 EM on the Cul-de-sac. (2) middle panel illustrates another linear

544 model of dissemination wherein a complex clonal population at the 
545 Rectum seed the cul-de-sac. Subsequently only a subset of these

546 clone travel to and populate the ovary. Finally, (3) illustrates a

547 differing model of spread wherein a complex clonal population at

548 the Rectum seed the cul-de-sac with all clones. Only a subset with

549 a single PIK3CA and KRAS altered clone break-off to the ovary

550 (or alternatively only a subset with these specific alterations can

551 colonize the ovary).

552

553 Tables

554 Table 1: Cohort Overview. Each cell shows the number of lesions

555 available and tested from the noted anatomical site/type (number

556 in brackets denote lesions were not tested in panel sequencing).

557 Blue cell $=$ shared alteration within case/between lesions marked

558 by blue cells in table (cells not in blue were uninformative/did not

559 show evidence of clonality with any other lesion from the given

560 case), Grey lines/cases were entirely uninformative (no mutation

561 detected in panel sequencing from any tested lesion).

562 Abbreviations: $\mathrm{DIE}=$ Deep infiltrating endometriosis, $\mathrm{EM}=$

563 superficial endometriosis, OMA= endometrioma

564

565 


\title{
566 References
}

\author{
567 \\ 568 1. Munksgaard PS, Blaakaer J. The association between \\ 569 endometriosis and ovarian cancer: a review of histological, \\ $570 \quad$ genetic and molecular alterations. Gynecol Oncol 124, \\ $571 \quad$ 164-9 (2012). https://10.1016/j.ygyno.2011.10.001 \\ 572 \\ 573 \\ 574 \\ 575 \\ 576 \\ 577 \\ 578 \\ 579 \\ 580 \\ 581 \\ 582 \\ 583 \\ 584 \\ 585 \\ 586 \\ 587 \\ 588 \\ 589 \\ 590 \\ 591 \\ 592 \\ 593 \\ 594 \\ 595 \\ 596 \\ 597 \\ 598 \\ 599 \\ 600 \\ 601 \\ 602 \\ 603 \\ 604 \\ 605 \\ 606 \\ 607 \\ 608 \\ 609 \\ 610 \\ 611 \\ 612 \\ 613 \\ 2. Zondervan $\mathrm{KT}$, Becker CM, Missmer SA. Endometriosis. N \\ Engl J Med 382, 1244-56 (2020). \\ https://10.1056/NEJMra1810764 \\ 3. Giudice LC. Clinical practice. Endometriosis. N Engl J Med \\ 362, 2389-98 (2010). https://10.1056/NEJMcp1000274 \\ 4. Eskenazi B, Warner ML. Epidemiology of endometriosis. \\ Obstet Gynecol Clin North Am 24, 235-58 (1997). \\ 5. Bulun SE. Endometriosis. N Engl J Med 360, 268-79 \\ (2009). https://10.1056/NEJMra0804690 \\ 6. Vercellini P, Vigano P, Somigliana E, Fedele L. \\ Endometriosis: pathogenesis and treatment. Nat Rev \\ Endocrinol 10, 261-75 (2014). \\ https://10.1038/nrendo.2013.255 \\ 7. Johnson NP, Hummelshoj L, Adamson GD, et al. World \\ Endometriosis Society consensus on the classification of \\ endometriosis. Hum Reprod 32, 315-24 (2017). \\ https://10.1093/humrep/dew293 \\ 8. American Society for Reproductive Medicine. Revised \\ American Society for Reproductive Medicine classification \\ of endometriosis: 1996. Fertil Steril 67, 817-21 (1997). \\ 9. Anglesio MS, Papadopoulos N, Ayhan A, et al. Cancer- \\ Associated Mutations in Endometriosis without Cancer. N \\ Engl J Med 376, 1835-48 (2017). \\ https://10.1056/NEJMoa1614814 \\ 10. Lac V, Verhoef L, Aguirre-Hernandez R, et al. latrogenic \\ endometriosis harbors somatic cancer-driver mutations. \\ Hum Reprod 34, 69-78 (2019). \\ https://10.1093/humrep/dey332 \\ 11. Suda K, Nakaoka H, Yoshihara K, et al. Clonal Expansion \\ and Diversification of Cancer-Associated Mutations in \\ Endometriosis and Normal Endometrium. Cell Rep 24, \\ 1777-89 (2018). https://10.1016/i.celrep.2018.07.037
}


medRxiv preprint doi: https://doi.org/10.1101/2021.04.12.21255355; this version posted August 25, 2021. The copyright holder for this preprint (which was not certified by peer review) is the author/funder, who has granted medRxiv a license to display the preprint in perpetuity.

It is made available under a CC-BY-ND 4.0 International license .

614

615

616

617

618

619

620

621

622

623

624

625

626

627

628

629

630

631

632

633

634

635

636

637

638

639

640

641

642

643

644

645

646

647

648

649

650

651

652

653

654

655

656

657

658

659

660

661

662

663

664
12. Piriyev E, Schiermeier S, Romer T. Coexistence of endometriomas with extraovarian endometriosis and adhesions. Eur J Obstet Gynecol Reprod Biol 263, 20-4 (2021). https://10.1016/j.ejogrb.2021.05.044

13. Exacoustos C, De Felice G, Pizzo A, et al. Isolated Ovarian Endometrioma: A History Between Myth and Reality. J Minim Invasive Gynecol 25, 884-91 (2018). https://10.1016/j.jmig.2017.12.026

14. Suda K, Cruz Diaz LA, Yoshihara K, et al. Clonal lineage from normal endometrium to ovarian clear cell carcinoma through ovarian endometriosis. Cancer Sci, (2020). https://10.1111/cas.14507

15. Montgomery GW, Giudice LC. New Lessons about Endometriosis - Somatic Mutations and Disease Heterogeneity. N Engl J Med 376, 1881-2 (2017). https://10.1056/NEJMe1701700

16. Lac V, Nazeran TM, Tessier-Cloutier B, et al. Oncogenic mutations in histologically normal endometrium: the new normal? J Pathol 249, 173-81 (2019).

https://10.1002/path.5314

17. Forbes SA, Bindal N, Bamford S, et al. COSMIC: mining complete cancer genomes in the Catalogue of Somatic Mutations in Cancer. Nucleic Acids Res 39, D945-50 (2011). https://10.1093/nar/gkq929

18. Khalique S, Naidoo K, Attygalle AD, et al. Optimised ARID1A immunohistochemistry is an accurate predictor of ARID1A mutational status in gynaecological cancers. J Pathol Clin Res 4, 154-66 (2018). https://10.1002/cjp2.103

19. Kobel M, Piskorz AM, Lee S, et al. Optimized p53 immunohistochemistry is an accurate predictor of TP53 mutation in ovarian carcinoma. J Pathol Clin Res 2, 247-58 (2016). https://10.1002/cjp2.53

20. Kobel M, Ronnett BM, Singh N, et al. Interpretation of P53 Immunohistochemistry in Endometrial Carcinomas: Toward Increased Reproducibility. Int J Gynecol Pathol 38 Suppl 1, S123-S31 (2019). https://10.1097/PGP.0000000000000488

21. Yong PJ, Talhouk A, Anglesio MS. Somatic Genomic Events in Endometriosis: Review of the Literature and Approach to Phenotyping. Reprod Sci, (2021).

https://10.1007/s43032-020-00451-9 
medRxiv preprint doi: https://doi.org/10.1101/2021.04.12.21255355; this version posted August 25, 2021. The copyright holder for this preprint (which was not certified by peer review) is the author/funder, who has granted medRxiv a license to display the preprint in perpetuity.

It is made available under a CC-BY-ND 4.0 International license .

665

666

667

668

669

670

671

672

673

674

675

676

677

678

679

680

681

682

683

684

685

686

687

688

689

690

691

692

693

694

695

696

697

698

699

700

701

702

703

704

705

706

707

708

709

710

711

712

713

714
22. Noe M, Ayhan A, Wang TL, Shih IM. Independent development of endometrial epithelium and stroma within the same endometriosis. J Pathol, (2018). https://10.1002/path.5082

23. Fassbender A, Rahmioglu N, Vitonis AF, et al. World Endometriosis Research Foundation Endometriosis Phenome and Biobanking Harmonisation Project: IV. Tissue collection, processing, and storage in endometriosis research. Fertil Steril 102, 1244-53 (2014).

https://10.1016/j.fertnstert.2014.07.1209

24. Rogers PA, Adamson GD, Al-Jefout M, et al. Research Priorities for Endometriosis. Reprod Sci 24, 202-26 (2017). https://10.1177/1933719116654991

25. Bulun SE, Wan Y, Matei D. Epithelial Mutations in Endometriosis: Link to Ovarian Cancer. Endocrinology 160, 626-38 (2019). https://10.1210/en.2018-00794

26. Artemova D, Vishnyakova P, Khashchenko E, et al. Endometriosis and Cancer: Exploring the Role of Macrophages. Int J Mol Sci 22, (2021). https://10.3390/ijms22105196

27. Olkowska-Truchanowicz J, Bialoszewska A, Zwierzchowska A, et al. Peritoneal Fluid from Patients with Ovarian Endometriosis Displays Immunosuppressive Potential and Stimulates Th2 Response. Int J Mol Sci 22, (2021). https://10.3390/ijms22158134

28. Oda K, Hamanishi J, Matsuo K, Hasegawa K. Genomics to immunotherapy of ovarian clear cell carcinoma: Unique opportunities for management. Gynecol Oncol 151, 381-9 (2018). https://10.1016/j.ygyno.2018.09.001

29. Lai CR, Hsu CY, Chen YJ, et al. Ovarian cancers arising from endometriosis: a microenvironmental biomarker study including ER, HNF1ss, p53, PTEN, BAF250a, and COX-2. J Chin Med Assoc 76, 629-34 (2013). https://10.1016/j.jcma.2013.07.008

30. Saavalainen L, Lassus H, But A, et al. Risk of Gynecologic Cancer According to the Type of Endometriosis. Obstet Gynecol, (2018). https://10.1097/AOG.0000000000002624

31. Chandler RL, Damrauer JS, Raab JR, et al. Coexistent ARID1A-PIK3CA mutations promote ovarian clear-cell tumorigenesis through pro-tumorigenic inflammatory 
715

716

717

718

719

720

721

722

723

72

725

726

727

728

729

730

731

732

733

734

735

736

737

738

739

740

741

742

cytokine signalling. Nat Commun 6, 6118 (2015).

https://10.1038/ncomms7118

32. Anglesio MS, Wang YK, Maassen M, et al. Synchronous Endometrial and Ovarian Carcinomas: Evidence of Clonality. J Natl Cancer Inst 108, (2016). https://10.1093/jnci/djv428

\section{Acknowledgements}

The authors thank all the study participants who contributed to this study. We further recognize the invaluable contributions of Prof.

Dr. Sara Y. Brucker for continuous support of international collaborations between the University Hospital Tübingen and the University of British Columbia, as well as PD Dr. Annette Staebler and the staff of the Institute of Pathology, University Hospital Tübingen for facilitating access to pathology archives.

Funding was provided by Canadian Institutes of Health Research (Early Career Investigator Grant in in Maternal, Reproductive, Child \& Youth Health to MS Anglesio). M Köbel received support through the Calgary Laboratory Services research support fund (RS19-609). MS Anglesio is funded through a Michael Smith Foundation for Health Research Scholar Award (18274) and the Janet D. Cottrelle Foundation Scholars program (managed by the BC Cancer Foundation). This project received technical and data management support from Calgary Laboratory Services and the Genetic Pathology Evaluation Centre (GPEC). GPEC receive core support from BC's Gynecological Cancer Research team (OVCARE), and the VGH+UBC Hospital Foundation. M Köbel 
743 was supported by internal research support (RS19-621). No

744 funder had any role in the study design, collection of data,

745 recruitment or participants, interpretation of results, manuscript

746 content, or decision to publish.

747

748 Compliance with ethical standards

749 Conflict of interest

750 The authors declare no competing interests.

751 Ethics approval/consent to participate

752 All work was done in compliance with the Canadian Tri-Council

753 Policy Statement (TCPS2 2018) on ethical conduct for research

754 involving humans. Effort to obtain written informed consent was

755 exercised for all patients. Specimen from non-contactable patients

756 (lost contact/deceased) treated more than 5 years before the start

757 of the study were included under institutionally approved waiver of

758 consent (Tübingen University Hospital Research Ethics Board).

759 Research Ethics Boards of all participating institutions approved

760 the study and use of materials.

$761 \quad$ Author contributions

762 MSA and SK designed the study and concept. THP, MG, BK, and

763 SK identified candidate patients and obtained consent. THP, VL,

764 and JS processed and enriched samples and conducted

765 molecular experiments. BTC, TMN and MK conducted and scored 
766 immunohistochemical experiments and reviewed/confirmed

767 endometriosis pathology. THP, JS, and MSA analyzed and

768 interpreted data. THP, AL, and MSA wrote the original draft. PJY,

769 BK, SK reviewed finding and all authors approved the draft

770 manuscript. Final draft was completed by AL and MSA.

$771 \quad$ Funding

772 This study received funding from the Canadian Institutes of Health

773 Research, the Michael Smith Foundation for Health Research, the

774 Janet D. Cottrelle Foundation, and the Calgary Laboratory

775 Services Research Support Fund.

776 Data availability

777 All data generated are available in this manuscript and

778 supplemental documents. Access to unprocessed/raw data from

779 the authors will be made available upon request, subject to REB

780 approval and applicable privacy laws. 
medRxiv preprint doi: https://doi.org/10.1101/2021.04.12.21255355; this version posted August 25, 2021. The copyright holder for this preprint (which was not certified by peer review) is the author/funder, who has granted medRxiv a license to display the preprint in perpetuity.

It is made available under a CC-BY-ND 4.0 International license .

A

Cases with Mutations in Endometriosis Lesions

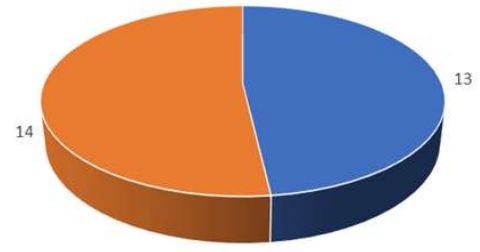

- Harboring mutation " Not Detected in Panel

C

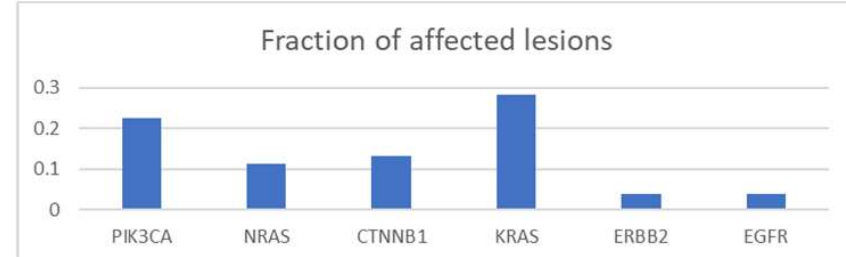

$E$

Fraction of affected cases/individuals

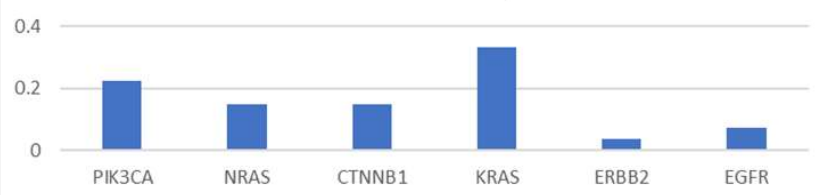

B
Mutations Detected in Panel Seq (\#)

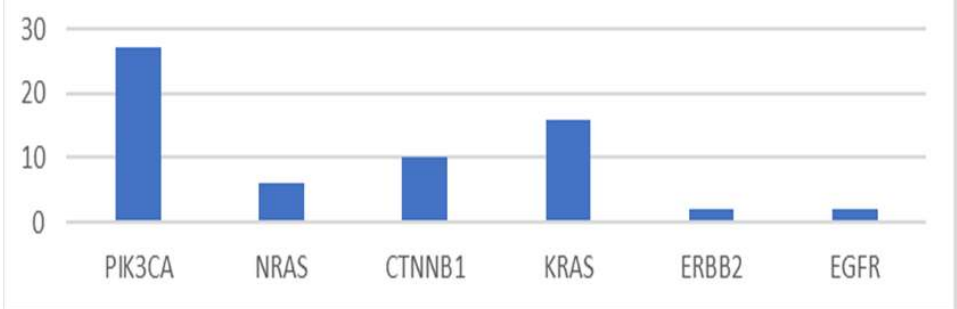

D

Fraction of affected lesions, split by lesion type

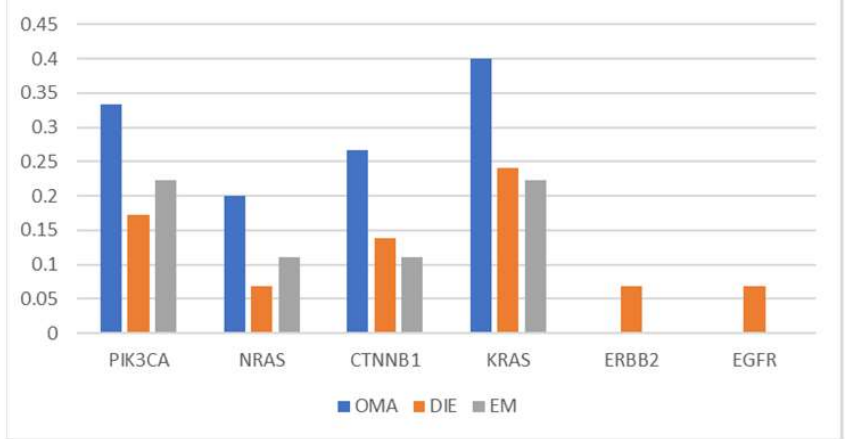


A

\begin{tabular}{|c|c|c|c|c|c|}
\hline \multicolumn{6}{|c|}{ Case 3 Detected Alterations } \\
\hline Gene & Hotspot & $\begin{array}{l}\text { OMA } \\
\text { Left ovary }\end{array}$ & $\begin{array}{l}\text { DIE (v) } \\
\text { Vagina }\end{array}$ & $\begin{array}{c}\text { DIE (c) } \\
\text { Cul-de-sac }\end{array}$ & $\begin{array}{c}\text { EM } \\
\text { Rectum }\end{array}$ \\
\hline PIK3CA & p.Glu545Lys & $\mathrm{x}$ & $x$ & & $\begin{array}{l}\text { Insufficient } \\
\text { size for } \\
\text { sequencing }\end{array}$ \\
\hline PIK3CA & p.Gly1049Ser & $\mathrm{x}$ & $\mathrm{x}$ & & \\
\hline PIK3CA & p.Arg88Gln & $x$ & $\mathrm{x}$ & & \\
\hline PIK3CA & p.Met1043lle & $\mathrm{x}$ & $\mathrm{x}$ & $\mathrm{x}$ & \\
\hline KRAS & p.Gly12Asp & $\mathrm{x}$ & $x$ & $\mathrm{x}$ & \\
\hline ERBB2 & p.Ser310Phe & $x$ & $\mathrm{x}$ & $\mathrm{x}$ & \\
\hline$\left\{\begin{array}{l}\text { ARID1A } \\
\text { IHC }\end{array}\right.$ & & $\begin{array}{l}\text { Loss in } \\
\text { atypical } \\
\text { endometriosis }\end{array}$ & & & $\begin{array}{l}\text { Hetero- } \\
\text { geneous } \\
\text { loss }\end{array}$ \\
\hline
\end{tabular}

B

(1)

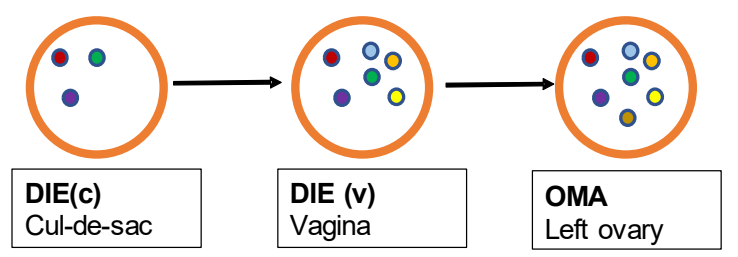

$(2)$
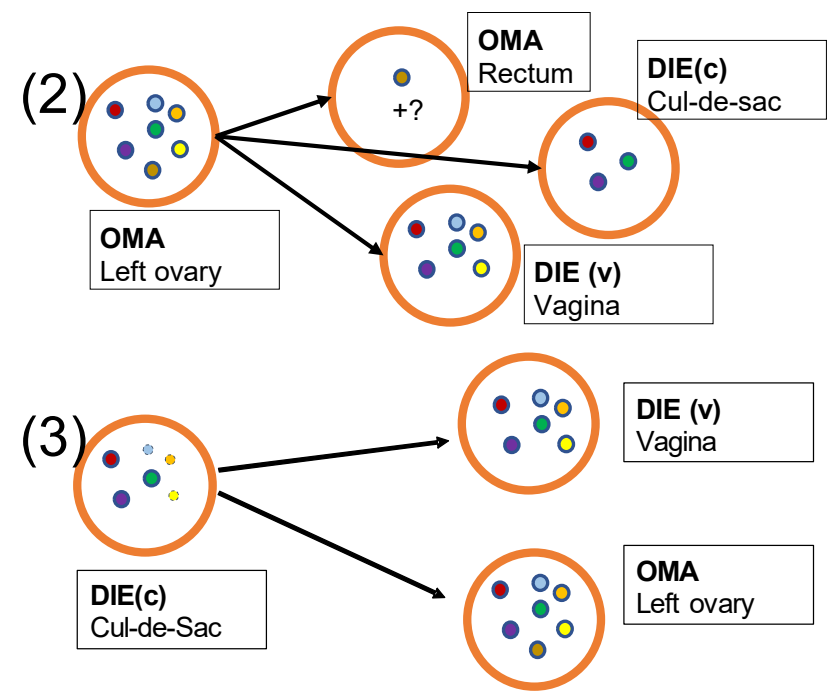

D

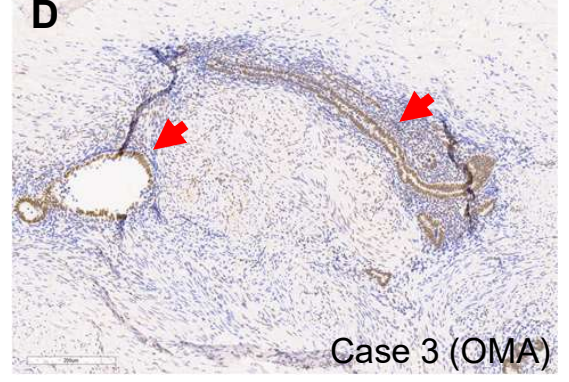

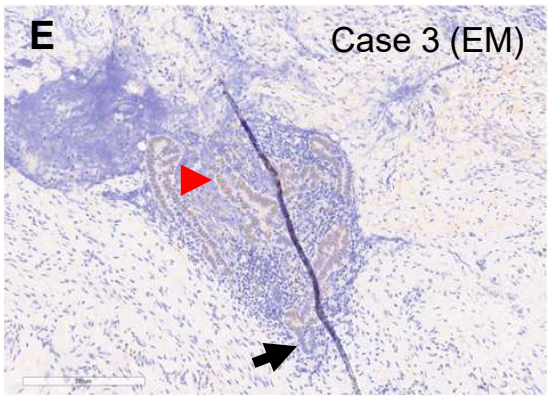


medRxiv preprint doi: https://doi.org/10.1101/2021.04.12.21255355; this version posted August 25, 2021. The copyright holder for this preprint (which was not certified by peer review) is the author/funder, who has granted medRxiv a license to display the preprint in perpetuity.

\section{It is made available under a CC-BY-ND 4.0 International license .}

A

\begin{tabular}{|c|c|c|c|c|}
\hline \multicolumn{5}{|c|}{ Case 4 Detected Alterations } \\
\hline Gene & Hotspot & $\begin{array}{c}\text { DIE } \\
\text { Rectum }\end{array}$ & $\begin{array}{c}\text { OMA } \\
\text { Left ovary }\end{array}$ & $\begin{array}{c}\text { EM } \\
\text { Cul-de-sac }\end{array}$ \\
\hline PIK3CA & p.Glu542Lys & $\mathrm{X}$ & & $\mathrm{X}$ \\
PIK3CA & p.Glu545Lys & $\mathrm{X}$ & & $\mathrm{X}$ \\
PIK3CA & p.Gly1049Ser & $\mathrm{X}$ & & $\mathrm{X}$ \\
PIK3CA & p.Met1043lle & $\mathrm{X}$ & $\mathrm{X}$ & $\mathrm{X}$ \\
\hline NRAS & p.Gly13Asp & $\mathrm{X}$ & $\mathrm{X}$ & $\mathrm{X}$ \\
\hline CTNNB1 & p.His36Tyr & $\mathrm{X}$ & $\mathrm{X}$ & $\mathrm{X}$ \\
CTNNB1 & p.Gly34Glu & $\mathrm{X}$ & & $\mathrm{X}$ \\
\hline KRAS & p.Gly12Asp & $\mathrm{X}$ & $\mathrm{X}$ & $\mathrm{X}$ \\
\hline
\end{tabular}

B

(1)

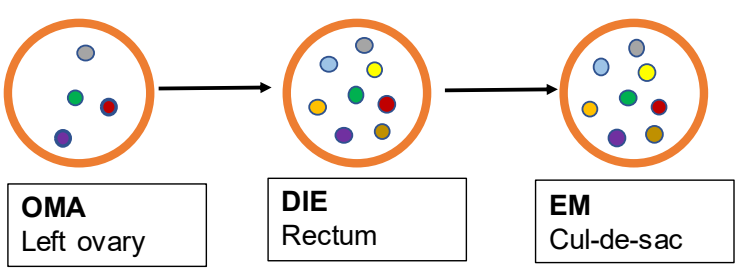

(2)

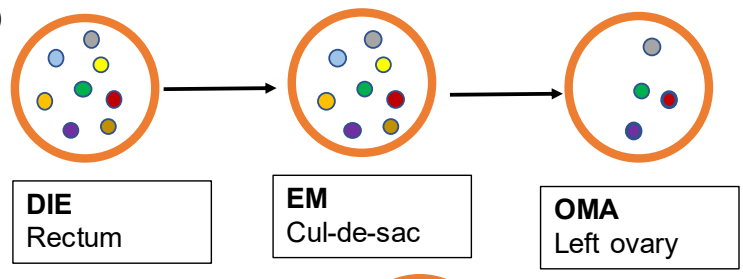

(3)

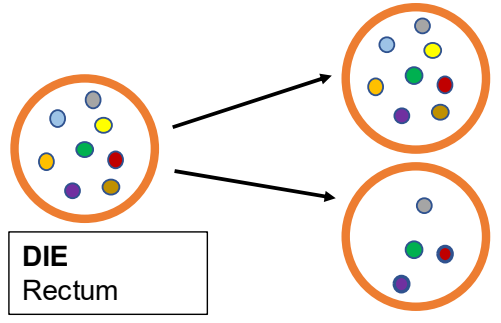

EM

Cul-de-sac

OMA

Left ovary 
Table 1 - Cohort overview

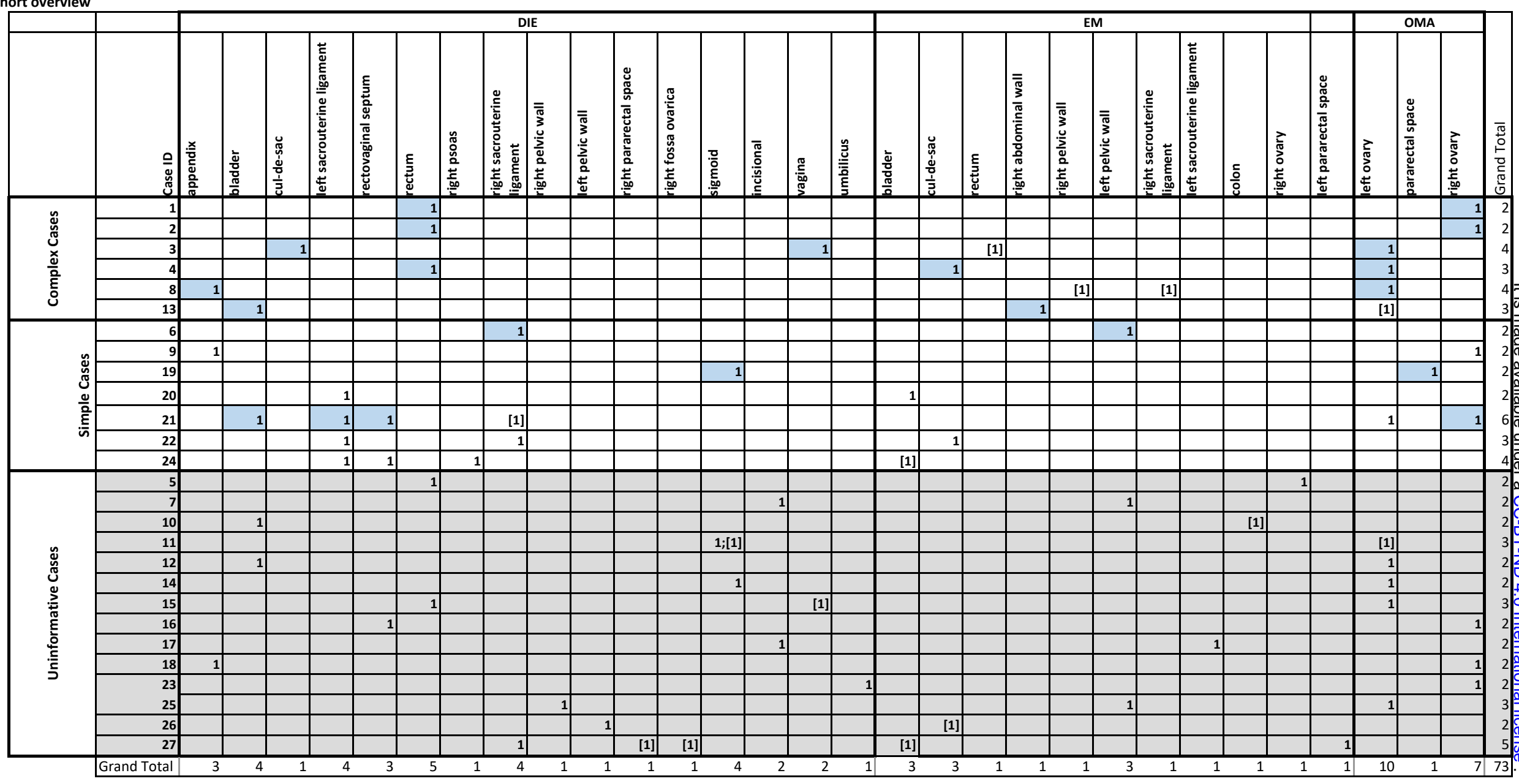

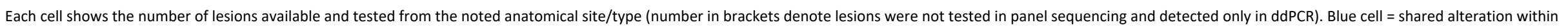

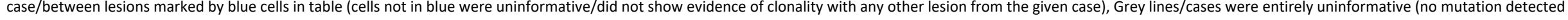
in panel sequencing from any tested lesion). Abbreviations: $\mathrm{DIE}=$ Deep infiltrating endometriosis, $\mathrm{EM}=$ superficial endometriosis, $\mathrm{OMA}=$ endometrioma 